\title{
TRATTA DI SCHIAVI TRA GENOVA E LA SPAGNA NEL SECOLO XV
}

\section{Geo Pistarino}

Uno dei commerci più fruttuosi sulla piazza di Genova nel secolo xv fu la tratta degli schiavi. Il volume di Domenico Gioffrè, Il mercato degli schiavi a Genova nel secolo XV, del 1971', ne ha dato un ampio quadro, esaminando il fenomeno sotto diversi punti di vista e fornendo inoltre, attraverso le tabelle in appendice, desunte da un vastissimo spoglio di documenti notarili dell'Archivio di Stato di Genova, un materiale notevole per ulteriori ricerche. In modo particolare queste tabelle consentono di avere sott'occhio il quadro dell'ambiente mercantile che opera in tale settore: ambiente formato in massima parte da genovesi, ma nel quale non mancano però anche commercianti di altre «nazioni», sia italiane, sia extraitaliane.

${ }^{1}$ D. GIOFFrE, II mencato degli schiavi a Genova nel secolo XV, Collana storica di fonti e studi, Genova 1971. Per il quadro complessivo della schiavitù medievale, ricordo i due ponderosi volumi di CH. VERLINDEN, L'esclavage dans l'Europe médiévale I, Brugge 1955, II, Gent 1977, il primo dei quali è dedicato esplicitamente alla penisola iberica. In particolare sulla schiavitù a Genova cfr. L. TRIA, "La schiavitù in Liguria (ricerche e documenti)», in Atti della Società ligure di storia patria LXX, 1947; G. PISTARINO, «Tra liberi e schiave a Genova nel Quattrocentom, in Anuario de estudios medievales I, 1964; Id., «Sul tema degli schiavi nel Quattrocento a Genovan in Miscellanea di storia 
La grande maggioranza degli acquirenti «esterni» sulla piazza di Genova proviene dalla penisola iberica, in particolare dalla Spagna: i più sono dell'area catalano-aragonese, o di quella biscaglina; alcuni pochi appartengono all'area castigliana. Ciò non può non destare qualche interrogativo dal momento che la penisola iberica, nel lungo progresso della Reconquista, tra paci e guerre, guerriglia e scorrerie, era essa medesima un serbatoio di rifornimento di «merce» umana. Ma la spiegazione del fenomeno della diversa frequenza di commercianti ispanici (catalanoaragonesi e biscaglini, da una parte, castigliani, dall'altra) sul mercato genovese, sta appunto nella sproporzione tra gli operatori ispanici del Nord (in grande maggioranza) e quelli del Sud (in forte minoranza).

La Castiglia rimase infatti sino all'ultimo, cioè sino alla caduta del regno moresco di Granada, in điretto contatto territoriale con l'area islamica. L'Aragona, con l'annessa Catalogna, ne venne invece separata, ad un dato momento, già nel secolo xıII, proprio in conseguenza del dilagare dei Castigliani verso il Mezzo-

ligure IV, Genova 1966; R. DELORT, "Quelques précisions sur le commerce des esclaves à Gênes vers la fin du xive siècle», in Mélanges d'archéologie ef d'histoire 78, 1966; G. BALBI, «La schiavitù a Genova tra i secoli XII e XIID, in Mélanges René Crozet II, Poitiers; A. HAVERKAMP, «Zur Sklaverei in Genua während des 12. Jahrhunderts", in Geschichte in der Gesellschaft. Festschrift K. Bosl, Stuttgart 1974; M. BALARD, «Remarques sur les esclaves à Gênes dans la seconde moitié du XIIle sièclen, in Mélanges d'archéologie et d'histoire LXXVIII, 1976; CH. VERLINDEN, «Le recrutement des esclaves à Gênes du milieu du xue siècle jusque vers $1275 \%$, in Fatti e idee di storia economica nei secoli XI-XX. Studi dedicati a F. Borlandi, Bologna 1977; G. PIS. TARINO, "Genova e la Sardegna nel secolo XII», in La Sardegna nel mondo mediterraneo. Atti del primo Convegno internazionale di studi geograficostorici, Sassari 7-9 aprile 1978, Sassari 1981; vol. II, pp. 33-125; J. HEERS, Esclaves et domestiques au moyen-âge dans le monde méditerranéen, Paris 1981; G. PISTARINO, "Schiave e schiavi sardi a Genova (secc. XII-XIII)", in Archivio Storico Sardo di Sassari VIII, 1982; M. BALARD, «Le minoranze orientali a Genova (secc. XIII-XV)m, in La storia dei Genovesi, Genova 1983, vol. III, pp. 71-90; L. BALLETTO, «Stranieri e forestieri a Genova: schiavi e manomessis, in corso di stampa negli Atti del Seminario internazionale di studio a Vilal di Mondeggi - Bagno a Ripoli, 4-8 giugno 1984. 
giorno della penisola. Sicché questi ultimi ebbero la costante possibilità di rifornimenti attraverso la situazione bellica e le occupazioni militari, nonché attraverso gli scambi commerciali con le terre islamiche confinanti; mentre i catalano-aragonesi trovarono più comodo, e probabilmente anche più conveniente sul piano economico, servirsi dei viaggi a Genova per effettuare gli acquisti necessari. Noto, come fatto concomitante e significativo, la quasi totale assenza di commercianti portoghesi, nel vasto movimento del mercato schiavistico genovese del secolo $\mathrm{Xv}$ : li si incontra soltanto un paio di volte, e sempre come venditori di schiavi mori o negri. Ed è naturale: il Portogallo aveva di fronte a sé, ed alle sue campagne di esplorazioni e di conquiste, i vasti spazi africani, sicché la situazione si pose qui in maniera notevolmente diversa.

Un alto numero di contrattazioni, in cui si trovano coinvolti in qualche modo gl'iberici, riguarda appunto in Genova gli schiavi mori, con prevalenza degli uomini sulle donne (21 contro 18 ): il che dimostra che un'area del rifornimento è proprio la Spagna del Sud, tanto più che la frequenza delle notizie, offerte dalle tabelle del Gioffrè, s'infittisce a partire dal 1458 e poi dal 1485, quando entra nella fase finale e risolutiva il conflitto granadino-castigliano, con la conquista cristiana di Alhama nel 1482 , la cattura di re Boabdil ed il trattato di Cordova nel 1483, la conquista di Álora e di Setenil nel 1484, le campagne militari di re Ferdinando nel 1485-86, la presa di Malaga nel 1487 con la riduzione in servaggio dell'intera popolazione, la conquista di Huéscar nel 1488, di Almeria nel 1489 ed infine l'ingresso dei Castigliani in Granada il 2 gennaio 1492. Esiste dunque una tratta di schiavi dalla Spagna meridionale a Genova e di qui all'area catalano-aragonese e biscaglina.

Questi schiavi, provenienti sia dal Nord-Africa sia dal territorio di Granada, non sono esclusivamente mori ed islamici: il riferimento, nei nostri documenti ogni tanto, al colore della pelle (olivegna, nera, bianca, laurina) indica la frammistione di razze che era tipica del regno granadino, la quale era anche frammistione religiosa. Caso significativo mi sembra quello di Isabella, mora alba, di 21 anni, venduta nel 1495 da Giovanni di Casti- 
glia, hispanus, al setaiolo Leonardo de Arziola: credo che si tratti appunto di una moresca di pelle chiara, già suddita dello scomparso regno dei $\mathrm{Nazarì}^{2}$. Un esempio ancora più sintomatico mi sembra quello della schiava Maria che nel 1487 ricorre ai Sindacatori per essere dichiarata libera, poiché afferma di non essere mora, ma hispana, cioè cristiana: era una cattolíca del regno di Granada $^{3}$ oppure l'oggetto di una voluta confusione?

Numerosi, nelle richieste dei mercanti catalano-aragonesi sul mercato di Genova, anche gli schiavi russi, con una nettissima prevalenza delle donne sugli uomini (almeno 29 su un totale di 37 ), ed i circassi ${ }^{4}$, con prevalenza del sesso femminile sul maschile (21 contro 14). Vengono poi, a notevole distanza, i tartari, su piano di parità tra maschi e femmine (11 contro 11), e gli abkhasi, con prevalenza del sesso femminile sul maschile (11 contro 2). Per le minori entità, troviamo 6 fra turchi e turche (4 contro 2), 5 bulgare, 2 gote, 2 guance o canarie, un'ebrea, una ungherese, un mingrelio, una greca, 7 tra uomini e donne senza indicazione di etnia. In totale: 166 unità $^{5}$.

Degli schiavi mori ci è notizia dal 1424 al 1497 , con isolate indicazioni nel 1424 ed una nel 1432 ed una specifica frequenza nella seconda metà del secolo. Per i russi, dopo alcuni accenni nel 1403 , le notazioni sono relativamente fitte dagli anni venti

${ }^{2}$ Sulle schiave more albe cfr. D. GiOFFRẺ cit., p. 140. Cfr. anche R. URBANI, "Genova e il Maghrib tra il ' 400 e '500 (nuovi documenti archivistici)", nel vol. Genova, la Liguria e l'Oltremare tra medioevo ed età moderna. Studi e ricerche d'archivio II, Genova 1976, pp. 198-199.

${ }^{3}$ Sulla presenza di cristiani-cattolici nel regno moresco di Granada nel secolo xv cfr. G. Pistarino, Presenze ed influenze italiane nel Sud della Spagna (secc. $X(1-X V)$, in corso di stampa.

4 Il Gioffrè ha considerato unitariamente i circassi e gli zichi, sotto la qualifica dei primi, mentre le due stirpi andrebbero più opportunamente considerate separatamente, in quanto gli zichi erano una distinta tribù, assai meno numerosa, apparentata ai circassi: CH. VERLINDEN, L'esclavage, cit., II, p. 486.

${ }^{5}$ Questi sono i dati che si ricavano dalle tabelle del Gioffrè, i quali vanno considerati come minimalí. Per i canari o guancí si tenga presente che il nome di guanci andrebbe applicato soltanto agli aborigeni di Tenerife, anche se poi si c̀ esteso, per convenzione, agli abitanti di tutte le isole (nella lingua madre degl'indigeni la voce «guanche» significa semplicemente «uomo»). 
agli anni sessanta, mentre già mancano negli anni settanta. Per i circassi, fatta eccezione per la vendita della tredicenne Lucia nel 1401 da parte di Tommaso Embriono a Pietro Gonsales de Ordieles de Hispania, s'incomincia a parlare nel 1412, con più fitto spessore tra gli anni venti e trenta e negli cinquanta e sessanta sino ai primi degli anni settanta, dopo di che non se ne trova più cenno. E per i tartari, dopo cinque notazioni tra il 1400 ed il 1404, e due nel 1417, troviamo qualche consistenza dagli anni venti ai cinquanta, poi due sole notizie, nel 1462 e nel 1466. I turchi s'incontrano in un caso nel 1415 e poi nel 1478-91; le bulgare, nel 1427 e nel 1463-66; le gote, nel 1416 e nel 1460; le guance o canarie, nel 1465 e nel 1468; l'ungherese nel 1450; il mingrelio, nel 1466; la greca, nel 1486; l'ebrea, nel 1494, probabile vittima dei provvedimenti antisraeliti dei Re cattolici.

In sostanza, il fatto, che risulta subito evidente, è l'addensarsi delle notizie sull'export degli schiayi orientali da Genova alla Spagna tra gli ultimi lustri della prima metà del secolo xv ed i primi della seconda metà. All'opposto, l'incremento delle presenze degli schiavi mori nel progresso della seconda metà del Quattrocento fa da contrappeso alla diminuzione numerica, sino alla scomparsa, degli orientali. Le ragioni del fenomeno sono già state indicate dal Gioffrè e non da lui soltanto, sicché non occorre insistervi. Un fatto peculiare è la presenza delle due guance o canarie, che si colloca nel quadro di una tratta a cui parteciparono anche i genovesi: richiamo qui l'attività di «Johanot Otobo de Mor, mercader jenoves" (secondo la grafia di uno scriba catalano), il quale nel 1494 trasportò un carico di 65 indigeni da Teneriffe a Valencia ${ }^{6}$.

Diamo qui, in ordine cronologico, l'elenco degli spagnoli che nel secolo XV risultano attivi sul mercato schiavistico genovese, quale si ricava dal complesso delle tabelle del Gioffrè:

${ }^{6} \mathrm{CH}$. VerLINDEN, «L'esclavage dans le centre et le nord de l'Italie continentale au bas moyen âge”, in Studia Historica Gandensia 127, 1970, p. 122. Johanot Otobo de Mor è forse un Giovannotto Ottobono de Mari. 
Antonio Feo di Barcellona $\quad 1400$

Pietro Gonsales de Ordiales o Ordieles

1401,1421 de Hispania, civis Cerexiis

Bernardo Casadachila mercante di Barcellona 1403

Giovanni Rame di Barcellona $\quad 1403$

Guarnardo Cessaberges mercante di Valencia 1403

Lodisio Fraxinet o Fraxineto di Valencia 1403

Giovanni Plat o Plates o Prates mercante 1403,1404 catalano di Barcellona

Iacopo o Giano Ferrerio o Jame Ferrerio o 1403, 1420, Ferrero, catalano di Barcellona o Valencia 1424, 1425,

1426,1427

Gonzales Sanches castigliano

1412

Iacopo Villa di Maiorca

1415

Pietro de Clerano, civis Maioricis, mercante 1415

Marco (de) Spil di Maiorca

1416,1417

Nicola Demeto mercante di Maiorca

1417

Lopez de Lam de Hispania $\quad 1418$

Pietro Roix, civis et habitator Sibilie, patrono $\quad 1418$ di nave

Bartolomeo di Valencia, imponerius

Jame Nirondo di Maiorca

1425

Bernardo Casaldayguila mercante catalano

Francesco Toriolo di Maiorca

Giovanni Garsia di Siviglia

Guglielmo Mir di Barcellona

1426

Giovanni Brondo catalano

Guglielmo de Puris di Maiorca

Michele Ferrerio catalano, mercante in Genova

Nicola Oliverio di Maiorca

Valentino Garigo di Barcellona

Violante di Valencia

Pietro Gavilla mercante di Maiorca

Pietro Siforteise di Maiorca

Bartolomeo Girones o Gizones catalano di Maiorca

Fererio Dalman catalano di Maiorca 
Giovanni de Ferreria de Sancto Anderi del regno 1433 di Castiglia

Giovanni de Valdesio del regno di Castiglia

1433

Bernardo Lorenzo catalano di Barcellona

1434

Gabriele Diomel catalano, patrono di nave

1434

Jame Fabreges di Barcellona

1434

Perez di Bilbao

1443

Bernardo de Alba catalano

1447

Giovanni Modrens di Barcellona

1447

Jame Bertram

Giorgio Veglio di Ibiza

1447

Raffaele Beso di Barcellona

1448

Bernardo Dala o Dale catalano

1448

1448,1449 ,

1450

Giovanni Re mercante catalano, Giovanni Ros mercante di Barcellona, Ianeto Res mercante

1448,1451 , catalano

Lodisio Manuele catalano

1450

Gonsalo o Gonzale Rois mercante catalano

1451,1452

Francesco Diez di Xeres fu Dego

1453

Michele Navarro biscaglino

1453

Martino Ochoa de Madaria biscaglino

1454

Alopes de Palm de Hispania, patrono di nave 1455

Domenico Albias di Tortosa

1456

Egidio Rois catalano

1456

Giovanni de Gibeleone de Hispania

1456

Diego de Basulto biscaglino

1457

Ferrando de Visnaga biscaglino

1457

Pasquale (de) Soldis de Belmeo biscaglino,

patrono di nave

Pietro de Deva biscaglino

1457

Pietro de Portu de Undaroa o Vindaroa

1457 biscaglino

Martino Sevalle o de Sobala biscaglino

1457,1460

Giovanni Ferrandes de Ermendua biscaglino

1459

Francesco de Ibiza de Valmar mercante

1459,1460 di Valencia 
Martino de Albulis o Arbulis de Belmeo del 1459,1460 regno di Castiglia biscaglino

Francesco Pedralbes di Tortosa mercante tolosano 1459, 1466

Pietro de Ascoeta o Aschoeta de Granicha 1460 biscaglino

Giovanni Geronense mercante di Maiorca 1460,1461

Matteo Viacamp o Viacamps, Delcagni di 1461,1462 , Tortosa mercante aragonese o catalano 1465,1467 , 1474

Giovanni de Astue de Lipusto biscaglino 1462

Giovanni Focoberto o Fontecoberto mercante di 1462, 1463, Maiorca

1465,1471

Emanuele Ibaroia catalano

1463

Guglielmo de Rat catalano 1463

Raffaele Mercader di Barcellona 1463,1464

Pietro Cagnisar mercante di Tortosa 1464

Sanchez de Cerculis mercante di Valencia 1464

Vidal o Lodisio Vidal di Maiorca 1464,1467

Galerano o Galcerano o Gerolamo Adret de 1465,1466 , Falceto mercante catalano 1469

Gabriele Marco catalano di Valencia 1465,1466 , 1469,1472 , 1473,1487

Jame de Sos, civis e mercante barcellonese 1468,1468

Dalmatio de Arra di Tarragona 1469

Giovanni Marco mercante catalano di Valencia 1470,1491

Giovanni de Sumelso patrono di Bilbao 1474

Bernardo Torres di Ibiza

Alonso Sanches 1476,1478

1477

Francesco Lampagies di Maiorca

Giovanni Roys de villa Dove regni Biscaie

Martino de Marcheta biscaglino 1482

Gabriele di Tolosa mercante catalano 1485 di Barcellona

Giovanni de Circulis catalano, procuratore di 1485 Simoneto de Belprato, legato del re Ferdinando Francesco Pedralbes catalano mercante di Tortosa 1486 
Lodisio Ropolli, commissario del re d'Aragona

Francesco Panario di Valencia

Giovanni di Castiglia hispanus

Sono dunque un centinaio di nominativi, più esattamente 97; ma questo è un numero che non fa testo, sia per l'aleatorietà della documentazione notarile pervenutaci, sia perché non siamo sicuri di talune identificazioni. Ad esempio, sono la medesima persona, o sono almeno due persone distinte, Iacopo o Giano Ferrerio, Jame Ferrerio o Ferrero catalano, ora detto di Barcellona ed ora di Valencia? E la stessa incertezza non vale anche per Giovanni Re mercante catalano, Giovanni Ros mercante di Barcellona, Ianeto Res mercante catalano?

I mercanti ispanici si susseguono pressocché ininterrottamente a Genova nel corso del Quattrocento. Abbiamo tre cesure nella serie cronologica, di qualche rilievo, tra il 1404 ed il 1412, tra il 1418 ed il 1424 , soprattutto tra il 1434 ed il 1443: il periodo che corrisponde alla battaglia di Ponza ed alla crisi tra la Repubblica di Genova, il Ducato di Milano e la Corona d'Aragona. $\mathrm{Va}$ ancora sottolineato il fatto che il numero delle presenze è pressappoco equivalente tra la prima e la seconda metà del secolo $\mathrm{xV}$, anzi con una leggera prevalenza in quest'ultima fase: il che, comunque, sta a dimostrare come, al contrario che per altri frequentatori «esterni», ad esempio i toscani', gl'iberici non abbandonassero mai il mercato genovese.

Alcuni di questi comercianti sono classificati semplicemente come catalani o come biscaglini. Nelle più specifiche designazioni dei catalani vengono innanzi tutto i barcellonesi, in numero

${ }^{7}$ Cfr. G. PISTARIno, Tratta di schiavi da Genova in Toscana nel secolo XV, in corso di stampa. 
di 16 ed i maiorchini, in pari numero; seguono i valenciani, in numero di 8; mentre, come si è detto, c'è un nominativo riferito ora a Barcellona ed ora a Valencia che può fare supporre sia un errore di designazione sia l'individuazione in due persone distinte. Sono in numero minore gli uomini di Ibiza, di Tortosa, di Tarragona, di qualche altra località. I biscaglini di siti diversi, tra cui Bilbao, Bermeo, Deva, nel regnum Biscaie, sono 16. Poco più di una mezza dozzina i castigliani, tra cui uomini di città di Santander, di Siviglia... Né manca chi è nativo di un luogo e viene classificato come mercante od anche cittadino di un altro: ad esempio, Francesco Pedralbes di Tortosa, mercante tolosano (penso a Tolosa di Biscaglia); Gabriele di Tolosa, mercante di Barcellona; Jame de Sos, civis e mercante barcellonese. Ed è significativa la doppia qualifizazione di Matteo Viacamp, mercante aragonese e mercante catalano.

La distribuzione geografica non è cronologicamente uniforme. Nella prima metà del secolo si tratta, nella grandissima maggioranza, di catalani, barcellonesi, maiorchini, valenciani. I mercanti di Ibiza entrano nel gioco nel 1448, i biscaglini nel 1453, quelli di Tortosa nel 1456, mentre di un mercante di Xeres si ha notizia nel 1453 e di uno di Tarragona nel 1469. L'area del mercato schiavistico iberico-genovese si allarga progressivamente; si fa, per così dire, più capillare nella seconda parte del Quattrocento rispetto alla prima.

Oltre una ventina dei nostri personaggi sono tecnicamente classificati come mercatores. Ma ci sono anche almeno cinque patroni di navi, e c'è un imponerius ${ }^{\sharp}$, Bartolomeo di Valencia, il quale appartiene al ceto artigianale. Ciò mostra come schiave e schiavi fossero una «merce» passibile di traffici occasionali, soggetta anche alle improvvise opportunità, e per la quale non occorreva competenza.

La metà circa di questi iberici compaiono in affari della tratta schiavistica a Genova una sola volta. Altri vi figurano due o più volte, in un medesimo anno od in anni diversi, sì che si

${ }^{8}$ Ritengo che si debba rettificare la voce imponerius, data nel regesto del documento delle tabelle del Gioffrè, in iuponerius. 
può giungere a considerarli, in alcuni casi, come frequentatori abituali della piazza, i quali non acquistano schiave e schiavi sempre per uso proprio, ma fungono anche da imprenditori, acquirenti di «merce» per la rivendita nel proprio paese.

Né mancano quelli che prendono dimora in Genova dandone specifica dichiarazione nei documenti notarili, come Pietro de Clerano catalano, civis di Maiorca, nel 1415; il catalano Michele Ferrerio nel 1427; il catalano Bernardo Dala nel 1449; il catalano Galerano Adret de Falceto nel 1466 e 1469; Jame de Sos di Barcellona nel 1466; Gabriele Marco di Valencia nel 1469; Dalmatio de Arra di Tarragona nel medesimo anno; Francesco Pedralbes di Tortosa nel 1485; Gabriele di Tolosa mercante catalano nel medesimo anno. Ma si può presumere che per alcuni il soggiorno fosse più lungo di quello che risulta negli atti, come nel caso di Gabriele Marco, o di Bernardo Dala che nel 1450 loca quale balia la circassa Agnese, ventiduenne, a Francesco Giustiniani per due anni. Oppure è supponibile che risiedessero a Genova per qualche tempo, anche altri di cui non viene data specificazione, come in qualcuno dei casi di Bartolomeo di Valencia, che nel 1424 loca per 6 anni la circassa Maddalena, trentaseienne, a Tommaso di Roma, magister schlolarum; del catalano Lodisio Manuele, che nel 1450 prende in affitto l'unghera Novella, venticinquenne, per 2 anni e mezzo da Bartolomeo da Passano; del catalano Egidio Rois, che nel 1456 prende in affitto la circassa Lucia, ventunenne, per tre anni da Antonio Usodimare; di Gabriele di Tolosa, mercante di Barcellona, che nel 1485 loca per 6 anni la mora Grazia, trentasettenne, a Cristoforo Centurione; del catalano Michele Boneto, che nel 1486 loca per 8 anni la diciottenne greca Caterina ad Antonio di Marco; di Francesco Panario di Valencia, che nel 1495 prende in sublocazione la schiava Maddalena da Galeoto Salvago; di Pietro Soler di Barcellona, che nel 1497 loca per 8 anni la mora Pellegrina, diciottenne, ad Andrea Dondo.

Residenti o non residenti a Genova, sono comunque trafficanti di rilievo Iacopo Ferrario (se si identificano in un unico personaggio le diverse specificazioni onomastiche), che effettua gli acquisti tra russi, circassi e mori; Bernardo Dala, che traffi- 
ca in schiave tartare e circasse ed è procurate di Giovanni Sul di Barcellona, armerius maior del re di Aragona, il che può in parte spiegare il suo successo; Giovanni Re (con la medesima riserva di cui sopra circa l'identità), il quale opera nel settore maschile fra i tartari, nel settore femminile tra russe e abkhase; Francesco Pedralbes di Tortosa, che prima commercia tra i soggetti tartari e russi, poi tra i mori ed i turchi; Matteo Viacamp di Tortosa, trafficante in soggetti russi, circassi, abkhasi e mori; Giovanni Focoberto di Valencia, attivo tra i russi, i circassi, gli abkhasi, i bulgari, i canari; Galerano Adret de Falceto, che compera soggetti di stirpe tartara, russa, bulgara, finanche ebraica; Gabriele Marco e Giovanni Marco di Valencia, rivolti al settore dei circassi, degli abkhasi, dei mori, dei canari. Né possiamo dimenticare Bernardo Torres e Pietro Tur di Ibiza, che acquistano esclusivamente schiavi mori, o il catalano Giovanni de Circulis, il quale nel 1485 compra due donne unitamente ad un bambino con un unico contratto.

Dati più precisi sull'entità della tratta Genova-Spagna ci sono forniti, per alcuni momenti, dai registri ufficiali del Comune genovese. Dal cartulare dell'Introitus floreni del 1413 apprendiamo che, in quell'anno, 27 catalani avviarono al mercato spagnolo 82 schiavi, 45 maschi, 30 femmine e 7 di cui non è specificato il sesso $^{10}$. Sono particolarmente impegnati nel commercio Francesco Laurentio, con l'acquisto di 13 schiavi; Nicola Demeto e Jame Ponte, con l'acquisto, ciascuno, di 11 soggetti; Pietro Blancha, proprietario di 6 mancipia; Francesco Montilio, proprietario di 5 donne ed un uomo; Guglielmo Girao e Giovanni Laurentio, proprietari, rispettivamente, di cinque e quattro soggetti" .

Il cartolare del carato del 1423 c'informa di otto navi che salpano da Genova verso le terre catalane, al comando, rispettivamente, di Pietro Vachez, Iacopo Gamba di Maiorca, Pietro Martinez, Martino Perez de Biscaglia, Marco Gambone da Palermo,

9 A proposito di questi personaggi cfr. anche D. GlofFrE, cit., p. 169.

${ }^{10}$ D. GIOFFRE cit., p. 167.

${ }^{11}$ D. GIOFFRÉ Cit., pp. 167-168: 
Cristoforo de Trinixio, Apollonio Burzario, Francesco Aloarde: su tutte sono imbarcati schiavi. Jame Ferrario ne possiede 24; Iacopo Brondo di Maiorca, 3; Giovanni Ochoa e Dego Rodriguez, uno per ciaseuno. Un terzo di questi schiavi è costituito da mori.

Il cartulare del carato del 1448 fornisce i nomi di sei catalani acquirenti di schiavi: fra gli altri Giorgio Noxilio, Giovanni Re, Andrea Sipur, Lodisio Murnela, Iacopo Beltrame. Più rari i nominativi compresi nel cartulare del Drictus Catalanorum del 1453 per quanto riguarda gli operatori spagnoli che esportano schiavi da Genova. Soltanto la nave di Joham Garcia ne imbarca due per un valore totale di 175 lire $^{12}$.

Vi sono, tra i mercanti iberici in Genova, figure di rilievo per grado sociale: il catalano Giovanni de Circulis, già citato, il quale, in veste di procuratore di Simone de Belprato, legato del re Ferdinando di Castiglia, nel 1485 compera da Enrico de Camilla un gruppo di tre schiavi (Francesca di 22 anni, Macorri di 17 con il figlio Ferrando) per 326 lire genovesi; Lodisio Ropolli, commissario del re d'Aragona, che nel 1491 vende a Nicola Franco da Lipari lo schiavo Cristoforo, di 25 anni, per 40 ducati.

Troviamo anche, come già accennato, due venditori portoghesi: Joham de Sponos che nel 1476 vende la quindicenne mora Isabella a Battista Sofia per 55 ducati, ed Alfonso Diez da Lisbona che nel 1487 vende la quattordicenne Francesca, Maura de Ginea, a Francesco d'Arquata (ritengo che si tratti di Arquata Scrivia), pro tali qualis est, per la più modesta somma di 30 ducati e mezzo ${ }^{13}$. Siamo nella seconda metà del Quattrocento, quando cioè Lisbona diventa una piazza importante per la tratta degli indigeni di colore.

Sarà opportuno ricordare che c'era anche un traffico diretto via mare su navi genovesi, fra le aree d'acquisto e le terre iberiche. Ad esempio, nel 1445 dodici schiavi ( 9 balabani e 3 donne), imbarcati a Pera sul naviglio di Pietro Embrono, viaggiano per

12 D. GIOFFrE cit., p. 168.

${ }^{13}$ Il contratto di compra-vendita di Francesca è registrato nelle tabelle del Gioffrè sia tra i mori sia tra i negri. Ritengo che sia più esatta quest'ultima collocazione. 
Maiorca. Un patrono, Cosma Dentuto, che compie il periplo da Rodi ad Alessandria, a Chio, alle Fiandre, all'Inghilterra, colloca qualche schiava a Maiorca ${ }^{14}$.

$$
* * *
$$

Nella grande maggioranza sono contratti di vendite, effettuate da genovesi a mercanti spagnoli per esportazione. Tuttavia gli iberici figurano non solo come acquirenti, ma talvolta come venditori, tanto ad acquirenti loro connazionali, quanto ad acquirenti genovesi o di altre «nazioni». La «merce» è composta esclusivamente di orientali sino ai primi degli anni settanta; poi quasi esclusivamente di mori e turchi.

Bernardo Casadachila barcellonese vende la tartara Caterina a Gaio da San Miniato nel 1403; Iacopo Ferrerio o Jame Ferrero catalano vende la russa Maddalena a Guarnardo Cessaberges di Valencia nel medesimo anno; Violante di Valencia vende la russa Maddalena al catalano Guglielmo de Puris nel 1427; Giovanni de Valdesio del regno di Castiglia vende il tartaro Iacopo a Giovanni de Ferreria de Sancto Anderi (Santander) nel 1433; Giovanni Re catalano vende la russa Caterina a Demetrio de Nigrono nel 1451; Giovanni Ferrandes de Ermendua, biscaglino, vende la schiava Elena, di cui non è indicata la stirpe, al genovese Nicola Ancona nel 1459; Giovanni Marco di Valencia vende la circassa Anna a Francheta Grillo nel 1470; Alonso Sanches vende il moro Giovanni ad Aspeto di Zoagli nel 1477; Lodisio Ropolli, commissario del re d'Aragona, vende il moro Cristoforo a Nicola de Franco da Lipari nel 1491; ancora Giovanni Marco catalano di Valencia vende la turca Lucia al setaiolo Bartolomeo Ricio nel medesimo anno; Ingho de Artago di Bilbao, patrono di barca, vende l'ebrea Santiagun a Tommaso Gropallo nel

14 D. GIOFFrê cit., p. 170 nota 10 . La voce «balabano» è di origine turca, sinonimo dell'arabo umamelucco" o schiavo-soldato. Indica gli schiavi originari delle rive settentrionali del Mar Nero, cioè russi, tartari, circassi, destinati all'esercito dei sultani mamelucchi d'Egitto. Per estensione si applicava anche ai maschi provenienti dalle medesime regione e destinati ad altri paesi che l'Egitto, senza alcuna implicazione militare: $\mathrm{CH}$. VERLINDEN, cit., II, pp. 346-347. 
1494; Giovanni di castiglia, hispanus, vende la mora alba Isabella a Leonardo de Arziola nel 1495; Iacopo Joham de Mais di Maiorca vende il moro Nicola a Damiano Assereto nel 1497.

Oltre agl'iberici, tra i venditori non genovesi, che con loro commerciano sul mercato schiavistico di Genova, troviamo nomi di varia provenienza: Antonio de Martis di Bonifacio nel 1415; una donna, Caracosa di Biassa, nel 1417; Carlo di Corvara e Gabriele di Busalla nel 1427; Iacopo di Prato e Domenico di Loreto nel 1428; Antonio di Precipiano nel 1449; Diego di Cremona nel 1453; il milanese Venturino Borromeo nel 1454; il milanese Iacopo Maldato e Costantino di Malta nel 1456; Clara di Moneglia e Rinaldo Pasturella di Siracusa nel 1457; Giorgio di Chiavari nel 1464; Cristoforo di Bargagli nel 1482; Federico de Varna di Palermo e Pietro de Petra nel 1486, ed altri ancora. Come si vede, si va dagli odierni sobborghi genovesi ai centri della Riviera, a Milano ed a Cremona, da un lato, alla Corsica, a Palermo, a Malta, dall'altro. Quello degli schiavi è un mercato in cui s'impegnano a Genova tutti coloro che appena possono, approfittando della libera piazza.

E' un fenomeno che trova un riscontro significativo, se si prendono in esame le professioni anche solo di una parte di coloro che trattano con gl'iberici. Tra i venditori genovesi e non genovesi ci sono uno speciarius (Colombano Rainucio da Bobbio) nel 1403; un calzolaio (Battista Garrono) nel 1427; un notaio (Tommaso di Levanto) nel medesimo anno; un faber $^{15}$ (Bartolomeo di Sestri Ponente) nel 1430; un lanaiolo (Simone di Reggio) nel medesimo anno; un setaiolo (Giovanni Badano) nel 1441; un sonator arpe (Giovanni di Bargagli) nel 1461; un macellaio (Giovanni Cabella) nel 1462; un merciaio (Battista di Moneglia) nel 1464; un barberius (Giovanni Merello) nel 1465; un coiraserius (Domenico di Cannobbio) nel 1466; un fabbro (Geronimo di Porrata) nel 1467; un bambaxerius (Francesco da Recco)

${ }^{15}$ Sul significato della voce faber a Genova cfr. G. PISTARINO, La civiltà dei mestieri in Ligura (sec. XII), in "Saggi e documenti II», Genova, Civico Istituto Colombiano, Studi e testi, Serie storica a cura di G. Pistarino, n. 3, 1982, pp. 9-74. 
nel 1469; un tintore di seta (Battista Perolerio) nel 1474; un taverniere (Antonio Vinciguerra) nel 1496.

Il numero maggiore degli operatori economici, che trattano con gl'iberici, è rappresentato però da membri di esimie famiglie di Genova. Ricordo Domenico Campofregoso nel 1403, Manuele Lomellini nel 1404, Alessandro Balbi nel 1409, Goffredo Fieschi nel 1415, Bianchina Doria nel 1416, Domenico de Mari nel 1424, Nicola Gentile e Battista Usodimare nel 1425, Raffaele Bracelli e Francesco Fatinante nel 1426, Manfredo de Guisulfis, Lodisio de Franchi e Bartolomeo Cattaneo nel 1427, Antonio Gentile nel 1430, Pancrazio Falamonica nel 1432, Giovanni Gregorio Stella da Taggia, Gottardo da Sarzana, cancelliere del Comune di Genova, e Battista de Fornari nel 1448, Paolo Battista Lercari nel 1449, Bartolomeo da Passano nel 1450, Antonio Usodimare nel 1456, Baldassare Usodimare nel 1457, Federico Cicala nel 1457 e nel 1460, Egidio Carmadino nel 1457 e nel 1463, Francesco Grimaldi, Andrea Doria e Guirardo Spinola nel 1460, Nicola Centurione nel 1464, Benedetto de Goano, Giacomo Sauli e Domenico Pallavicino nel 1465, Mariettina Spinola nel 1466, Goffredo Lercari nel 1467, Raffaele Doria nel 1468, Battista Salvago nel 1469, Mariettina Imperiale nel 1472, Giorgio de Marini e Giuliano de Nigro nel 1473, Giambattista de Mari nel 1479, Cipriano de Fornari nel 1484, Carlo Spinola nel 1485 , Luca Panigarola nel 1489.

Possiamo concludere che Genova è un mercato schiavistico nel quale operano genovesi e forestieri; che tra i venditori vi sono professionisti ed artigiani; che, tuttavia, la grande parte del commercio si trova nelle mani delle maggiori famiglie della città, operandovi anche le donne. Ed abbiamo sott'occhio i nomi di una serie di personaggi che durante l'intero corso del secolo xV intrattennero rapporti commerciali, attraverso la tratta degli schiavi - ma certo non sempre soltanto attraverso questa-, con uomini di affari catalano-aragonesi, biscaglini, castigliani.

Complessivamente gl'iberici impegnano nella compera di schiavi sul mercato genovese, con 135 contratti, non meno di lire 14.619 soldo 1 e denari 4 , oltre a 120 lire di «paghe», fiorini 91 , ducati 534, ducati d'oro larghi 29. Dalle vendite, con 15 con- 
tratti, ricavano non meno di lire 1354 soldi 10 , oltre a ducati 120 e mezzo. Genova è dunque per loro soprattutto una piazza per acquisti. Considerate per singole etnie le somme di cui sopra si ripartiscono come segue:

\begin{tabular}{|c|c|c|c|c|}
\hline . & Acquisti & & Vendite & \\
\hline$x^{2}$ & $\frac{\mathrm{N}}{\text { contratti }}$ & Importo & $\begin{array}{l}\text { N. } \\
\text { contratti }\end{array}$ & Importo \\
\hline Russi & 31 & $\begin{array}{l}\text { lire } 3965, \text { s. } 2, \text { d. } 8 \\
\text { duc. } 29 \text { oro larghi }\end{array}$ & 3 & lire $392, \$ .10$ \\
\hline Circassi & 30 & $\begin{array}{l}\text { lire } 3548, \text { s. } 7 \\
\text { duc. } 45 \text { oro }\end{array}$ & 2 & lire 292 \\
\hline Tartari & 21 & $\begin{array}{l}\text { lire } 1881, \text { s. } 9 \text {, d. } 8 \\
\text { fior. } 91 \\
\text { duc. } 70\end{array}$ & 2 & lire 160 \\
\hline Mori & 24 & $\begin{array}{l}\text { lire } 1723 \\
\text { "paghe» } 120 \\
\text { duc. } 279\end{array}$ & 5 & $\begin{array}{l}\text { lire } 310 \\
\text { duc. } 78\end{array}$ \\
\hline Abkhasi & 11 & lire 1630 & - & - \\
\hline Bulgari & 5 & lire 767 & - & - \\
\hline Etnia ignota & 4 & lire 380 & - & - \\
\hline Turchi & 4 & $\begin{array}{l}\text { lire } 245 \\
\text { duc. } 135\end{array}$ & 1 & lire 200 \\
\hline Goti & 2 & lire 277 , s. 2 & - & - \\
\hline Canari & 2 & lire 202 & - & - \\
\hline Mingreli & 1 & duc. 50 & - & - \\
\hline Negri & - & - & 1 & duc. 30 e $\frac{1}{2}$ \\
\hline Ebrei & - & - & 1 & duc. 12 \\
\hline
\end{tabular}


Ma non si tratta soltanto di semplici compra-vendite. Il quadro, che si ricava dalle tabelle del Gioffrè, è più ricco e complesso, sia in generale sia nel settore specifico dei rapporti genovesiispanici. Sappiamo, ad esempio, nel 1417, che la tartara Anna, ventiduenne, appartenente al mercante maiorchino Nicola Demeto, è stata catturata sulla nave, su cui era imbarcata per il trasferimento a Maiorca, ed è stata venduta a Sassari; che nel 1432 Francesco Metastasio rilascia atto di procura a Geronimo Grimaldi perché venda a Barcellona gli schiavi Barak ed Aberacoman, de progenie barbarorum, entrambi di 36 anni; che nel 1452 agisce in Genova Diego di Cremona quale procuratore di Gandisalo de Sernantes di Siviglia per la vendita della schiava mora Marina di ventisette anni, che egli cede al biscaglino Giovanni Ferrando de Ermendua.

Abbiamo già accennato ad esempi di donne date o prese in affitto. Talvolta al termine della locazione la schiava avrà diritto alla libertà: sarà questo il futuro, ad esempio, della circassa Lucia, data in affitto da Antonio Usodimare ad Egidio Rois nel 1456 per tre anni, e della mora trentasettenne Grazia, concessa per sei anni da Gabriele di Tolosa a Cristoforo Centurione nel 1485. Si operano cambi: come quello trattato nel 1487 fra Manuele Macono e Gabriele Marco di Valencia per gli schiavi mori Giorgio, di 15 anni, e Caterina, di 25 , con un conguaglio di 28 palmi di camocato. Si stipulano assicurazioni per il trasporto della «merce»: come quella che nel 1456 Giovanni de Gibeleone de Hispania stipula per lire 300 , al $3 \%$, per i suoi due schiavi che devono essere trasferiti da Genova a Maiorca sulla nave di Oliverio Calvo.

C'è chi non vuole o non può spendere molto oppure cerca di combinare gli affari parte in proprio e parte in società. Nel 1454 la russa Maria, di 30 anni, viene acquistata dal biscaglino Martino Ochoa de Madaria pro tali qualis est, cioè - diremmo noia scatola chiusa, per la somma di 100 lire, notevolmente inferiore ai prezzi correnti. Nel 1460 i biscaglini Martino de Arbulis de Belmeo e Pietro de Ascoeta de Granicha acquistano in società la trentottenne russa Margherita per sole 38 lire, ma nel contempo Pietro de Ascoeta si assicura la proprietà del quindicen- 
ne russo Iacopino per 29 ducati d'oro. Richiamo anche il caso, già citato, della mora Francesca, venduta nel 1487 da Alfonso Diez di Lisbona a Francesco di Arquata pro tali qualis est.

Un esempio di rapida speculazione sulla «merce» mi sembra quello del catalano Giovanni Marco che il 15 settembre 1491 compra da Raffaele Sanguineto la turca Lucia, di 16 anni, per 125 lire e la rivende già il 28 settembre al setaiolo Giovanni Ricio per 200 lire. D'altronde non è un caso isolato, perché operazioni consimili, seppure rare, si riscontrano in altri settori del mercato schiavistico.

Una situazione giuridicamente ed economicamente notevole, anche se rientra in una casistica non eccezionale, è quella della schiava Margherita, abkhasa, di 18 anni, la quale, nel 1443, insieme con altra schiava, viene data in accomendacione, al quarto del profitto, da Gaspare Doria al nizzardo Giovanni Litardo per la vendita in Maiorca: accomendacio del tutto peculiare, trattandosi di merce vivente che richiede spese di mantenimento e di trasporto, presenta rischi per malattia o morte dell'oggetto, è soggetta a variazioni caratteriali.

Sono relativamente frequenti i casi di affidamento, cioè di schiave e schiavi che vengono consegnati ad una terza persona per la vendita su mercato esterno, per lo più nei Paesi catalani. $\mathrm{Nel} 1457$ la trentunenne Caterina è affidata, per la vendita extra Ianuam, da Federico Cicala al biscaglino Pietro de Deva; nel medesimo anno un'altra schiava trentunnene viene consegnata da Egidio Carmadino al biscaglino Pietro de Portu de Vindaroa per la vendita a Maiorca; nel 1464 la circassa Margherita è data da Nicola Centurione al maiorchino Vidal per la vendita a Maiorca; nel 1478 gli schiavi turchi Macomer e Demetrio sono affidati da Lodisio de Camulio di Caffa al socio Bernardo Veneroso per la vendita in Catalogna.

Non mancano le controversie, come quella che nel $1453 \mathrm{si}$ dibatte tra il biscaglino Michele Navarro, da un lato, ed Andrea Pasano, dall'altro, sulla liceità del negozio di vendita di due schiavi russi; Anna, di 18 anni, e Giovanni, di 15.

Non sempre la «merce» è rassegnata ad essere considerata come tale. Nel 1492 la schiava Lucia fugge, mentre sta per essere con- 
dotta per la vendita sul mercato di Ibiza. Sappiamo che la vendita a padroni catalani ed il trasferimento in paesi catalani erano aborriti dagli schiavi, che li consideravano una sorta di punizioni e che come tali venivano talvolta minacciati, dai proprietari genovesi ai propri mancipia, in caso di cattiva condotta. Più volte nei contratti di vendita s'incontra la clausola con la quale l'acquirente s'impegna a non rivendere il proprio schiavo a catalani: nel 1441 Giuliano Colombano, che richiede la restituzione dello schiavo russo Giorgio, fuggito e rifugiatosi presso il vescovo di Tortona, deve impegnarsi, per riottenerlo, a non cederlo ad un catalano ${ }^{16}$. L'esistenza in Catalogna - e non a Genova- dell'amministrazione generale della «garde des esclaves") per l'assicurazione contro la fuga dei mancipia rappresenta un fatto significativo ${ }^{17}$.

La grandissima maggioranza di queste donne, per non dire la totalità, che sono vendute ad acquirenti iberici, professa la religione cristiana nel credo cattolico, come dimostra il loro nome personale: il che significa che ha già compiuto il passo che le inserisce più facilmente nel contesto del mondo occidentale.

I nomi femminili più largamente usati per queste schiave sono quelli del comune repertorio: innanzi tutto Lucia, Caterina, Margherita, Maddalena, Maria; poi, Marta, Sofia, Anna, Cristina, Elena, ed anche Agnese, Anastasia, Antonia, Cita, Demetria, Diana, Melica, Novella. Tra le more i nomi di Grazia, Isabella, Marina, Macorri, Pellegrina spiccano a sé - oltre ad alcuni degli appellativi sopra citati-, nella tradizione più tipicamente ispanica. Il quale fenomeno appare ancora più evidente nel settore dei nomi maschili, nel quale, oltre tutto, è maggiore la percentuale degli appellativi non cristiani. Nel quadro complessivo prevale il nome di Giorgio; seguono Giacomo, Martino, Giovanni, Venturino; troviamo poi Andrea, Bastiano, Cristiano, Francesco, Lanzaroto, Robindo, Rolandino. I mori presentano gli appellativi di Abderacoman, Ali, Alonsiho, Amet, Bark, Calem, Cristoforo, Ferrando, Gaspare, Ianico, Joham, Lino, Pietro, Tom-

16 D. GiOFFrề cit., p. 20.

17 D. GIOFFRE cit., p. 89. 
masino, una parte dei quali sono significativi sia della tradizione islamica sia di quella iberica. Per i turchi: Demetrio e Macomer.

Domenico Gioffrè ha rilevato «che nell'ultimo trentennio l'interesse di Catalani quali acquirenti degli schiavi sul mercato genovese tende ad affievolirsi. Nel decennio 1471-1480, infatti, gli aragonesi appaiono come compratori in 11 contratti su 38 , la partecipazione al negozio, quindi è pari al $29 \%$ circa. Nella decade seguente, le contrattazioni sono 58 e gli spagnoli figurano in otto soltanto: la loro domanda scende al $14 \%$ del totale. Nell'ultimo decennio, infine, i loro acquisti sono quasi inconsistenti: due soltanto su un totale di 71 compravendite. Più di frequente ora gl'iberici si presentano essi stessi come offerenti di schiavi ${ }^{18}$. E l'Autore dà le spiegazioni di questo fenomeno, collegato alla chiusura del mercato orientale in seguito alle conquiste turche, alle lotte ispaniche contro l'Islam, alle spedizioni cristiane in Marocco ed in Algeria, al dominio castigliano sull'arcipelago delle Canarie.

Si restringe la varietà della merce. Nella prima metà del secolo, come si è già accennato, si contrattano in buon numero i soggetti orientali, che gradualmente scompaiono tra gli anni sessanta e settanta, emergendo i mori, i quali, insieme con i turchi, restano pressocché gli unici ad essere commerciati dalla fine degli anni settanta in poi, con qualche rara eccezione per la presenza di persone di altra stirpe. La «merce» orientale diventa rara e costosa; quella occidentale corre a più basso prezzo. Un decreto del governo della Repubblica genovese del 26 aprile 1501, nel ribadire il divieto di matrimonio tra una schiava ed un servo all'insaputa del padrone della donna, stabilisce per i contravventori una penale di 350 lire, se la schiava «fuerit ex illis que ex Oriente veniunt»; di 250 lire se la serva è una mora, perché le more «minori precio emuntur" ${ }^{19}$.

${ }^{18}$ D. GIOFFrÉ cit., p. 169.

19 D. GIOFFRE cit., p. 141. 
Occorre tuttavia tenere presente che il fenomeno di decrescenza, illustrato dal Gioffrè, riguarda gli ultimi decenni del secolo. Nell'intero corso del Quattrocento, invece, poiché le contrattazioni sono particolarmente intense in alcuni lustri prima ed alcuni lustri dopo la metà del secolo, la seconda metà non solo presenta una regolarità di presenze iberiche sul mercato non inferiore, nel complesso, alla prima, e non solo annovera un buon numero di grossi imprenditori, ma anche mostra un impegno finanziario iberico nel commercio schiavistico in Genova non più basso, globalmente, anzi più alto, rispetto a quello della prima metà (si tenga presente, ad ogni modo, il fenomeno dell'incremento dei prezzi). I dati che si ricavano dalle tabelle del Gioffrè, per numero di contratti e per movimento di capitali in acquisti ed in vendita da parte iberica, relativamente alle singole etnie della «merce» ed al primo e secondo periodo, sono significativi: 
N.

contratti
N.

contratti Importo

\section{Etnia ignota}

I n. 1

II n. 2

I n. 1

II n. 4

Importo

lire 100

lire 280

Turchi

\section{lire 120}

lire 125
Goti

I n. 1 lire 107 , s. 2

II n. 1 lire 170

Canari

I -

II n. 2

duc. 135

n. 1 lire 200
I-

II n. 1

Negri

I -

Ebrei

I-

Russi

Circassi

Tartari

Mori

Abkhasi

I n. 5

II n. 6

Bulgari
I n. 17 lire 1961, s. 12 , d. 8 duc. 29 oro larghi

II n. 14 lire 2003, s. 10

I n. 13 lire 1282, s. 7

II n. 17 lire 2266

I n. 15 lire 1241 , s. 9, d. 8 fior. 91

II n. 6

lire 640

duc. 70

I n. 1 lire 70

II n. 23 lire 1653

"paghe" 120

duc. 279
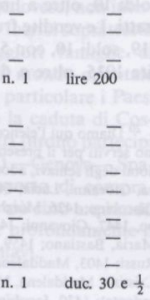

n. 1 duc. 12

n. 2 lire 87 , s. 10

n. 2 lire $\mathbf{3 0 5}$

n. 1 lire 72

n. 1 lire 220

n. 2 lire 160

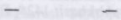

n. 5 lire 310 duc. 78

I n. 1 lire 140

II n. 4 lire 627

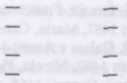


Complessivamente abbiamo quindi che $\mathrm{i}$ capitali impegnati dagli iberici sul mercato genovese per acquisti di schiavi nella prima metà del secolo XV non furono inferiori a lire 5702 , soldi 11 , denari 4 , oltre a fiorini 91 e ducati d'oro larghi 29, con 55 contratti; nella seconda metà, non furono inferiori a lire 8916 , soldi 10 , oltre a lire di «paghe» 120 e ducati 534 , con 80 contratti. Le vendite fruttarono, nella prima metà, non meno di lire 319 , soldi 10 , con 5 contratti; nella seconda metà, non meno di lire 1035 , oltre a ducati 120 e $\frac{1}{2}$, con 11 contratti ${ }^{20}$.

${ }^{20}$ Diamo qui l'elenco dei documenti delle tabelle del Gioffrè, di cui ci siamo serviti per il presente lavoro, secondo la classificazione per etnie, anni e nomi degli schiavi, adottata dall'Autore: Tartari: 1400, Martino; 1403, Caterina, Giovanni, Lucia; 1404, Cristiana; 1415, Marta; 1417, Melica, Anna; 1425, Giacobino: 1426, Margherita, Caterina, Margherita; 1427, Caterina; 1433, Iacopo, 1447, Giovanni; 1448, Andrea, Valentino; 1449, Iacobino; 1457, Marta, Maria, Bastiano; 1459, Valentino, Venturino; 1462, Antonina; 1466, Marco; Russi: 1403, Maddalena, Diana, Lucia; 1425, Giorgio; 1426, Cristiano; 1427 , Cristina, Maddalena, Margherita; 1428; Maria, Maria (forse la stessa della precedente); 1430, Iacobino, Caterina; 1434, Caterina, Caterina; 1443, Aspertino; 1448, Anna; 1449, - Margherita; 1451, Caterina, Marta; 1453, Anna, Margherita; 1454, Maria; 1457, Lucia, Antonia; 1460, Margherita, Iacopo, Caterina; 1463, Anastasia; 1464, Caterina; 1465, Maddalena, Maria; 1466, -; 1468, Sofia; 1469, Lucia, Margherita; Circassi: 1401, Lucia; 1412, Sofia; 1416, Sofia; 1418, Caterina; 1420, Martino; 1421, Marta; 1424, Maddalena; 1425, Giorgio; 1427, Maddalena, Giorgino, Giorgino, Margherita; 1430, Lanzaroto; 1432, Giorgio, Lanzarotto; 1450, Agnese; 1452, Anna; 1455, Maddalena; 1456, Martino, Lucia; 1457, Giorgino, Rolandino; 1459, Caterina, Caterina (la stessa della precedente); 1460, Giorgino, Valentino; 1462, Lucia; 1464, Margherita, Giorgio, Marta, Lucia; 1465, Elena; 1469, Anastasia; 1470, Anna; 1471, Francesco; 1474. Cita; Abkhasi: 1426, Elena; 1430, Margherita; 1434, Lucia; 1443, Margherita; 1448, Maddalena ed il figlio Martino, Lucia; 1459, Lucia; 1460, Valentino; 1462, Martino; 1467, Caterina, Lucia; 1468, Maria; 1482, Franceschina; Mingreli: 1466, Robindo; Mori: 1424, Iacopo; 1432, Barak e Abderacoman; 1453, -; 1459, Marina; 1461, Giacomino, Ali; 1463, Tommasino, Caterina; 1465, Anna; 1466, Maddalena; 1472, Caterina, Pietro; 1473, Caterina, Alonsiho; 1474, Giorgio; 1476, Isabella, Ioham; 1477, Giovanni; 1478, Anna, Ioham; 1479, Lucia; 1485, Grazia, Francesca e Macorri col figlio Ferrando, lanico, Lino; 1486, Gaspare; 1487, Maria, Caterina e Giorgio, Francesca (classificata anche tra i Negri); 1489, Calem e Amet; 1491, Cristoforo; 1494, Lucia; 1495, Isabella; 1496, Giovanni; 1497 , Nicola, Pellegrina; Negri: 1487, Francesca (classificata anche tra i Mori); Canari: 1465, Caterina; 1468, Caterina; Greci: 1486, Caterina; Bulga- 
Lo stato di tensione, di frequente rumor d'armi, di non pace e non guerra, che caratterizzò i rapporti tra la Repubblica di Genova e la Corona d'Aragona nel secolo xv, non influi in modo incisivo e determinante sui rapporti economici delle due aree; non fece diminuire in modo sensibile la presenza dei catalano-aragonesi sul mercato schiavistico genovese; non interruppe i contatti degli acquirenti, o venditori, iberici soprattutto con gli operatori commerciali delle maggiori famiglie di $G^{-n} v^{21}$. I genovesi funsero da tramite fra il mondo orientale e le terre della Corona d'Aragona, in modo particolare i Paesi catalani ${ }^{22}$, sino ad una ventina d'anni dopo la caduta di Costantinopoli in mano turca. Poi gli uni e gli altri furono partecipi del commercio triangolare: Spagna (Castiglia) - Genova- Spagna (Aragona-Catalogna), nel quale il rapporto di compravendita si andò gradualmente invertendo. E ciò sino a quando la scoperta dell'America venne a modificare profondamente il quadro internazionale nel mercato degli schiavi.

ri: 1427, Ellena; 1463, Elena; 1465, Margherita, Margherita; 1466, Demetria; Turchi: 1415, Caterina; 1478, Macomer e Demetrio; 1479, Venturina; 1484, Lucia; 1491, Lucia, Lucia (la stessa della precedente): Ungheresi: 1450, Novella; Ebrei: 1494, Santiagun; Goti: 1416, Sofia; 1460, Lucia; Senza indicazione di razza: $1447,-; 1456$, due schiavi da trasportare a Maiorca; 1457, Caterina, una schiava; 1459, Elena; 1495, Maddalena.

${ }^{21}$ Elenchi delle maggiori famiglie genovesi del Tre-quattrocento, in J. HeErs, Gênes au XVe siecle, Paris 1961; D. Gioffrè cit., p. 74; M. BALARD, La Romanie génoise (XIre-début du Xve siècle), Genova-Roma, 1978, p. 524; B.Z. KeDAR, Mercanti in crisi a Genova e Venezia nel '300, ediz. italiana, Roma 1983, pp. 200-201.

${ }^{22}$ Sottolineo anche la presenza dei biscaglini che nel secolo XV non fu limitata, in Italia, a Genova: cfr. C. TrasselLI, «Sui biscaglini in Sicilia tra Quattro e Cinquecenton, in Mélanges Escole Française de Rome, 1973. 